\title{
A support environment for the disclosure of heterogeneous systems documentation
}

\author{
D.H. Fijma, T.F. Verhoef, G.M. Wijers \\ ID Research \\ Kastanjelaan 4, 3833 AN Leusden, The Netherlands. \\ Tel. +3133951416, Fax. +3133945845. \\ E-mail: \{DFijma, DVerhoef, GWijers\}@idr.iaf.nl
}

\begin{abstract}
It is difficult to support the reuse of system components satisfactorily. This holds in particular for organisations in which responsibility for systems and system components is distributed over the organisation and in which many different systems documentation techniques are used. For those organisations, it is important to dispose of a support environment for the disclosure of heterogeneous systems documentation, which is characterised by flexibility and distribution. The requirements of such support environments are discussed. Based on these requirements the concepts and functionality of a prototype of such an environment are presented. Finally, it is discussed how different types of systems documentation from various sources can be handled by the prototype.
\end{abstract}

\section{Keywords}

Systems documentation, information distribution, decentralisation, reuse.

\section{Introduction}

Reuse of system components (Biggerstaf, 1989) has been widely recognised as a means to improve productivity of IT departments and, by consequence, to control the costs of systems development and especially of systems maintenance. In practice however, reuse of system components has proven to be a difficult goal to achieve, due to many different reasons. In this article, we point out two of those issues and deal with them: 
- Lack of standards If an organisation lacks well defined standards for the documentation of the systems developed and their components, many different techniques are used. This hinders the reuse of system components across organisation units.

- Decentralisation of responsibility While being advantageous in other aspects, an organisation structure with a high degree of decentralisation poses problems with respect to reuse. Responsibility for systems and system components is distributed over the organisation. Efficient communication of the documentation of available components becomes an important issue.

For organisations which are characterised by these two elements and which are willing to stimulate reuse of system components at the same time, it is important to dispose of a support environment for the disclosure of heterogeneous systems documentation in a way that is transparent with respect to the structure of and responsibility for the documentation contained in it.

In the literature, different environments have been described that support these requirements partially, e.g. (Arnold, 1987), (Brorsson, 1991), (Helm, 1991), (Garg, 1990). The flexibility of the environments described is not high enough for our needs. They offer only one, fixed, information model, to support a specific type of user documentation. Also, some of the environments support only a specific system development technique.

Naturally, the issues of flexibility and distribution in relation with systems documentation are discussed in literature. The environments described in (Freitag, 1994) and (Constantopoulos, 1992) both offer a variable document structure that can be used to define the specific documentation forms needed. Distribution is offered in the environment discussed in (van Oosterom, 1993). The particular combination, however, of the requirements of flexibility and distribution on an environment for the disclosure of systems documentation, has not been dealt with satisfactorily.

As an alternative to using specialised support environment for systems documentation, CASE tools could be considered. CASE tools that support flexibility are available (so-called CASE shells). Controlled distribution is a weaker point of CASE tools. As far as this is supported, the tools lack possibilities to communicate documentation across different organisational units, particularly if these groups use different techniques for systems documentation. The current generation of support environments does not support distribution and flexibility satisfactorily at the same time.

The problem situation sketched above has shown to be actual in a large Dutch governmental organisation. In the IT department of this organisation, reuse of system components is one of the major goals to be achieved as part of the overall strategy. Therefore, the organisation has initiated a project in which a prototype has been developed to demonstrate the functional possibilities and restrictions on a support environment for decentralised and flexible maintenance of heterogeneous systems documentation.

In section 2 , we identify the exact requirements such an environment should meet. In section 3 , we discuss the concepts underlying such an environment, and the functionality of a prototype that fulfils these requirements. In section 4 , it is discussed how systems documentation from different sources can be handled in the environment so that it supports both the concrete documentation type at hand and an abstract, reuse maximising meta-model. 
To conclude, in section 5, we discuss the lessons from the design of the environment and its benefits for future work.

\section{Requirements}

The two most important aspects of the requirements of the support environment pointed at are:

- Distribution. The distribution of systems documentation is needed to enable the reuse of components throughout the organisation.

- Flexibility. In the organisation, many different tools and techniques for system development are used. The environment must be able to deal with the resulting variety of systems documentation.

Distribution as meant here is logical distribution. We do not require the data to be physically stored in a distributed fashion. Naturally, from an implementation point of view, this may be a well suited option. Here, it is more important that the ownership of the systems documentation is distributed. In the envisioned environment, there is no centralised responsibility for the administration of some particular variety of documentation. If, for example, the set of entities is documented for various systems, these sets are owned and maintained by the organisational group that is responsible for the system concerned

Flexibility is needed because of the wide variety of types of systems documentation. The structure, or meta-model, according to which the documentation is to be stored, must therefore not be fixed. The environment must be able to operate with the different metamodels related to different tools and techniques in use, both current and future. On the other side, flexibility should be controlled. After all, the information stored should enable the reuse of system components across a diverse organisation. The abstraction level of the structure of documentation must therefore be chosen carefully. If too abstract a meta-model is chosen, the documentation retrieved from the system does not offer enough information to enable actual reuse. If the meta-model is too concrete, the documentation will only be of use to persons or groups of persons that use the same techniques and tools as the owner of the information. Ideally, the environment offers means for retrieving the same documentation on different levels of abstraction.

The required functionality of the support environment is illustrated in figure 1 . At the basis of the environment we find a document management facility. Around this kernel, specific functionality is partitioned over the different types of users of the environment. We distinguish three groups of users: system developers, system managers, and the controller(s) of the environment itself. To support these different types of users in the presence of the flexibility sketched, the environment should offer personalised work spaces. A user must be able to state the source and type of the systems documentation he is interested in, according to which structure or meta-model the documentation is viewed and which tools are used to access that documentation. In the remainder of this section, more specific requirements to the envisioned environment are discussed, for each of the three target audiences individually. 


\begin{tabular}{|c|c|c|}
\hline \multicolumn{3}{|c|}{ User authorisation } \\
\hline $\begin{array}{c}\text { Developer } \\
\text { Edit } \\
\text { Browse } \\
\text { Import } \\
\text { Export } \\
\text { Query }\end{array}$ & $\begin{array}{c}\text { Manager } \\
\text { Query } \\
\text { Report }\end{array}$ & $\begin{array}{c}\text { Controller } \\
\text { Configure } \\
\text { Quality } \\
\text { Control }\end{array}$ \\
\hline \multicolumn{3}{|c|}{ Communication } \\
\hline \multicolumn{3}{|c|}{ Document Management } \\
\hline
\end{tabular}

Figure 1 Functionality of the support environment.

\section{Support for system developers}

The system developer has to be supported by powerful tools to store and retrieve information He needs easy access to the environment, preferably directly from his desktop workstation The tools offered for retrieving information must support the following two tasks.

Firstly, the developer needs a query mechanism for finding reusable system components Many different types of conditions on such components must be expressible, like their principal function, implementation platform and possible interfaces.

Secondly, it is the task of the developer to explore the exact properties of the system components found earlier. For this task, one does not only need information on the selected component itself, but also on related components. This is, for example, important while exploring the possibilities for interfacing the selected component with other components.

Developers probably maintain systems documentation outside the environment, either by using some CASE-tool or manually. The effort of maintaining the documentation within the support environment must be minimised by offering interfaces between the developers' native documentation tools and the support environment. It must be prevented that a developer has to enter the same information twice during the development process.

\section{Support for managers}

Managers need less detailed information on system components than developers. Instead of detailed information on a single component, this type of users need a means for generation of summarising reports. Some of these reports are needed periodically, for example to gain insight in the growth of the number of available reusable system components the support environment offers. 


\section{Support for the environment controller}

The controller of the support environment does not manage or own the systems documentation stored. However, he is responsible for maintaining the meta-information of the support environment. For managing this information, the controller needs tools for creating and configuring users, groups of users and the meta-model according to which information is stored.

\section{User type independent support}

Two requirements are illustrated in figure 1 that are independent of the type of user. Firstly, the environment has a mechanism for authorisation.

The support of (electronic) communication is the second user independent requirement. Providing an easy way for communication between the different users of the support environment is important for the co-ordination of the disclosure of the distributed systems documentation. Preferably, the services provided are integrated with the existing systems for electronic mail.

The development of the environment according to the requirements above and its introduction in the organisation has several consequences. Beyond the technological consequences related to the development of a tool, we recognise both organisational and methodical consequences. Organisational consequences are considered to be important. The introduction of the environment aims at the maximisation of reuse. People will have to be stimulated to use the support environment and to reuse system components.

Equally important are the methodical consequences: how is the systems development process to be organised and, as a more direct consequence for the support environment, according to which meta-model is the systems documentation stored from various sources?

In this article, we focus on the environment itself. We discuss a prototype of an advanced support environment for the disclosure of heterogeneous systems documentation.

\section{A prototype}

A prototype of the envisioned support environment has been built in Smalltalk/V for Windows. The prototype was meant mostly as a vehicle for researching the possibilities and requirements for the support environment itself. The prototype further provides an illustration to future users of the possibilities to meet the requirements and the clearness of the concepts used.

First, we show how the various requirements discussed above are translated into a series of concepts the prototype deals with. Also, we give an overview of the actual functionality it presents its users.

\subsection{Concepts}

The concepts underlying the environment are discussed subsequently. Firstly, the concepts which are related to the core of the environment: object management, are discussed. After that, the concepts which are related to the requirements of distribution, flexibility, and coordination are presented 


\section{Object management}

The basic functionality of the prototype is to manage information in the form of systems documentation. Therefore, at the center, we identify an object management system (OMS). This OMS keeps track of the various classes or types of elements identified in the systems documentation to be stored.

The concepts implementing the OMS are illustrated in figure 2. Each circle in the figure presents a concept. The straight lines represent relations between concepts. Arrows denote a sub-type relation between concepts. As the figure illustrates, the OMS maintains information at two levels. At type level it keeps track of a set of object types. At instance level, it controls the actual objects, which are instances of a certain object type. An object is a single unit of systems documentation that is stored and manipulated in the prototype. For example, we could create object types such as 'table' and 'column'. Typical instances of these types would be 'school' or 'student' for the object type 'table' and 'address' and 'date' for 'column'.

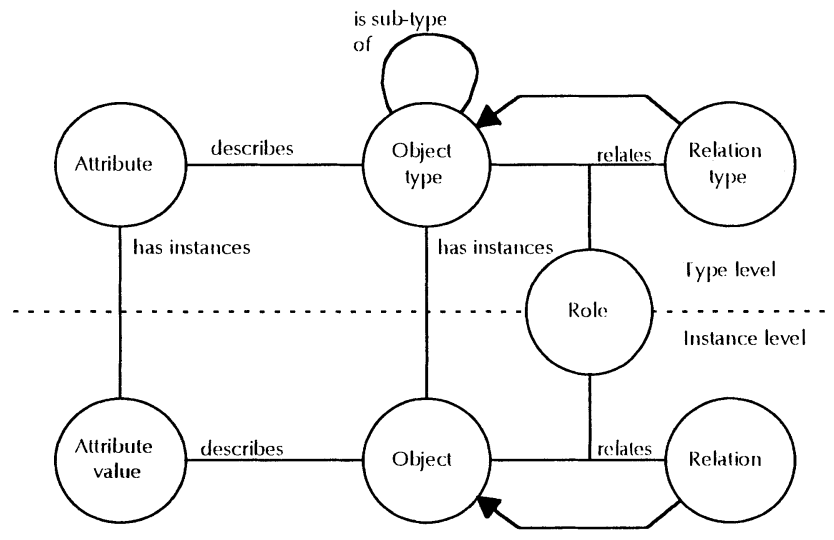

Figure 2 Structure of the Object Management System.

Attributes are related to object types. They constitute the basic values that describe instances of object types. As figure 2 illustrates, the OMS supports sub-typing. Any object type can be a sub-type of any number of object types. For example, we could create the object type 'information element' that is a super-type of both 'table' and 'column'. Information that is maintained for both tables and columns, can be handled uniformly through this generalising construct. Super-types are not abstract. That means, super-types can have instances that are not instances of any of its sub-types. This prevents the need for unwanted over-specialisation in the case of incomplete information. For example, in the absence of the knowledge whether something is a table or a column, we could store that something as an 'information element', without making unjustified assumptions about its actual concrete type.

Relations between object types are registered through relation types, like 'table has column' in the example above. Instances of relation types are relations, like 'school has address'. Because relation types are a special kind of object types (and relations a special kind of objects), everything that is possible with object types also applies to relation types. Most 
notably, relation types can have attributes. For example, the relation type 'student is registered at school' could have the attribute 'year of registration'.

The cardinality of relations is not restricted. A relation type can be associated with any number of object types. The concept of Role is used to keep track of the roles each object type plays in the relation type. The definition of roles is to be made clear at both the type and instance level.

\section{Distribution}

Directly related to the object management system a concept is identified that implements the basic requirement of distribution as formulated in section 2: context. 'Contexts' implement the distribution of ownership and responsibility for the systems documentation stored. This concept is illustrated in figure 3 . A context is a grouping mechanism by which responsibility can be given explicitly to project teams and departments in the organisation. Users have access to certain contexts. If an actual user belongs to a certain group in the organisation, he will have access to the context that represents that group. Objects, and thus systems documentation, are owned by a context. That means that the responsibility for maintaining information contained in an object lies with (the members of) a context. Objects can only be created, changed and deleted by their owners. Objects are not owned by individuals directly. Information on individual users is included only to control access to the environment and the contexts defined.

A context can have two kinds of relations with other contexts. Both these relations are used to control the distribution of information throughout the organisation. Firstly, a context can give access to its information to other contexts. In this way, a group can give other groups read-only access to its documentation. Secondly, a context can be a sub-context of another context, giving rise to a hierarchy of contexts. Sub-contexts inherit properties of their supercontext: if an user has access to a context, it has access to the sub-contexts of that context. Note that the 'is sub-context of' relation implies the 'has access to' relation.

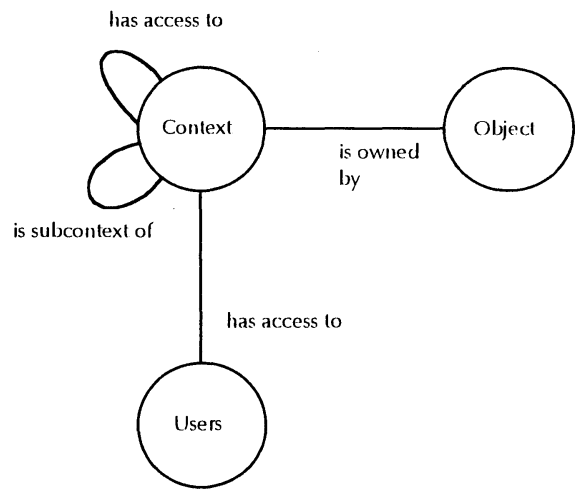

Figure 3 Contexts, access and ownership. 


\section{Flexibility}

The general requirement of flexibility is realised by the concepts of view and browser. A view controls what information is visible from a certain context and how that information is presented to the users within that context. Browsers are the actual tools used to access the systems documentation stored. The concepts of views and browsers are illustrated in figure 4 .

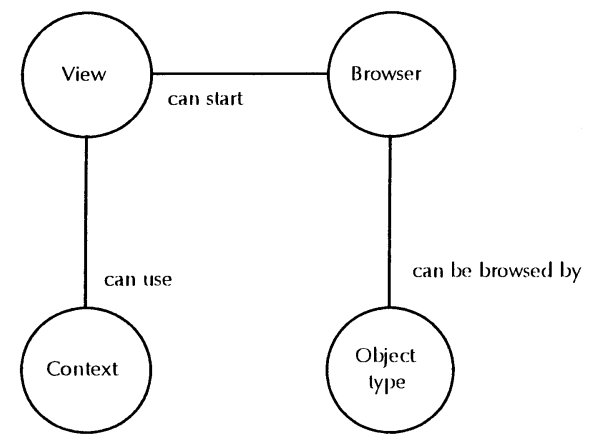

Figure 4 Views and browsers.

A browser is defined for a specific object type, for example a brower can show tables, or entities, or processes, etc. A browser can be used to create, delete or change instances of its related object type. Also, browsers implement the querying capabilities of the prototype. The functionality of browsers is discussed into more detail later. Here, it is important to understand that a browser is fit only to access the instances of one certain object type (and its sub-types). In the design of the browser it is determined which associated attributes and relation types of the object type are visible in the browser. A view is a set of browsers. The controller of the environment can configure the various contexts so that they can use the views they need for their documentation jobs.

\section{Co-ordination}

The last concept discussed here implements the requirement of co-ordination. The prototype offers the possibility to exchange electronic mail between users and contexts. Primarily, the mail system is based on users. However, it is possible to send a message to all users that have access to a certain context. The concept of electronic messages is illustrated in figure 5 .

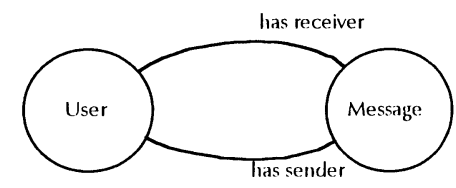

Figure 5 Electronic messages between users. 


\subsection{Functionality}

Three types of users were identified in section 2. In the prototype as it is, this has led to two different working environments. Developers and managers are served by the same working environment, that satisfies both their needs. For the controller of the environment, a separate working environment has been designed. As this environment presents rather straightforward tools for the configuration of the environment, the functionality of these tools is not discussed further here. For the developer's and manager's working environment we first discuss the facilities to set specific working options. After that, the basic functionalities with regard to browsing, navigation and querying are discussed.

\section{The entry of the system and how to set specific options}

The user has to login to the system. After having logged in using his user name, his password and the name of the context he wishes to enter, the user is presented the 'session window'. The session window is the main window for developers and managers. In the session window, see figure 6, the user can set options for his working environment and start browsers to access the systems documentation contained in the support environment.

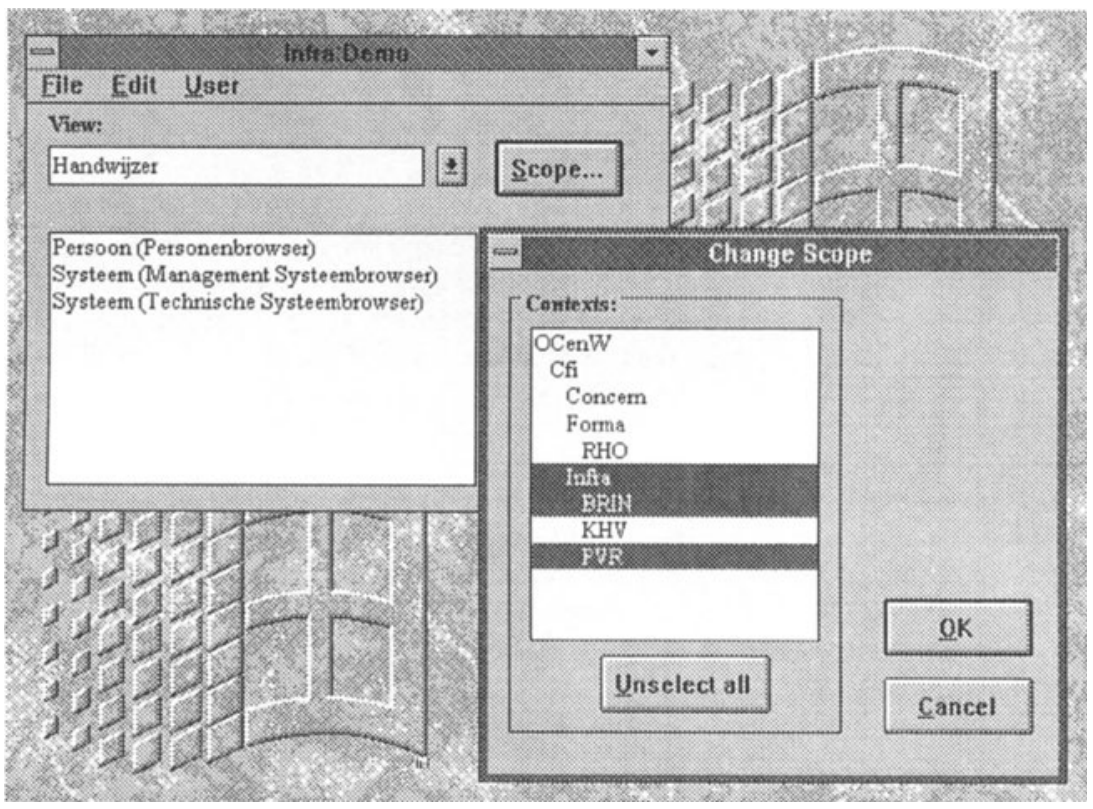

Figure 6 The session window and the definition of the scope.

After selecting the appropriate view, the user can start one or more browsers from the presented list. The functionality offered by the browsers will be discussed below. Access to other contexts than the one used to login is provided via the concept of scope. The scope is 
basically a set of contexts. A browser only displays those objects that are element of the contexts within the scope defined (see also figure 6). Naturally, to add a context to the scope, the context selected at login must have access to that other context (as discussed in section 3.1).

The session window offers access to the electronic mail system. This option starts a relatively standard mail tool, that is not discussed further in this article.

\section{Browsing and navigating}

Browsers implement the functionality to browse, change, retrieve and query the systems documentation. At any time, many browser can be open, thus giving access to different types of information at the same time. An example of an browser is shown in figure 7.

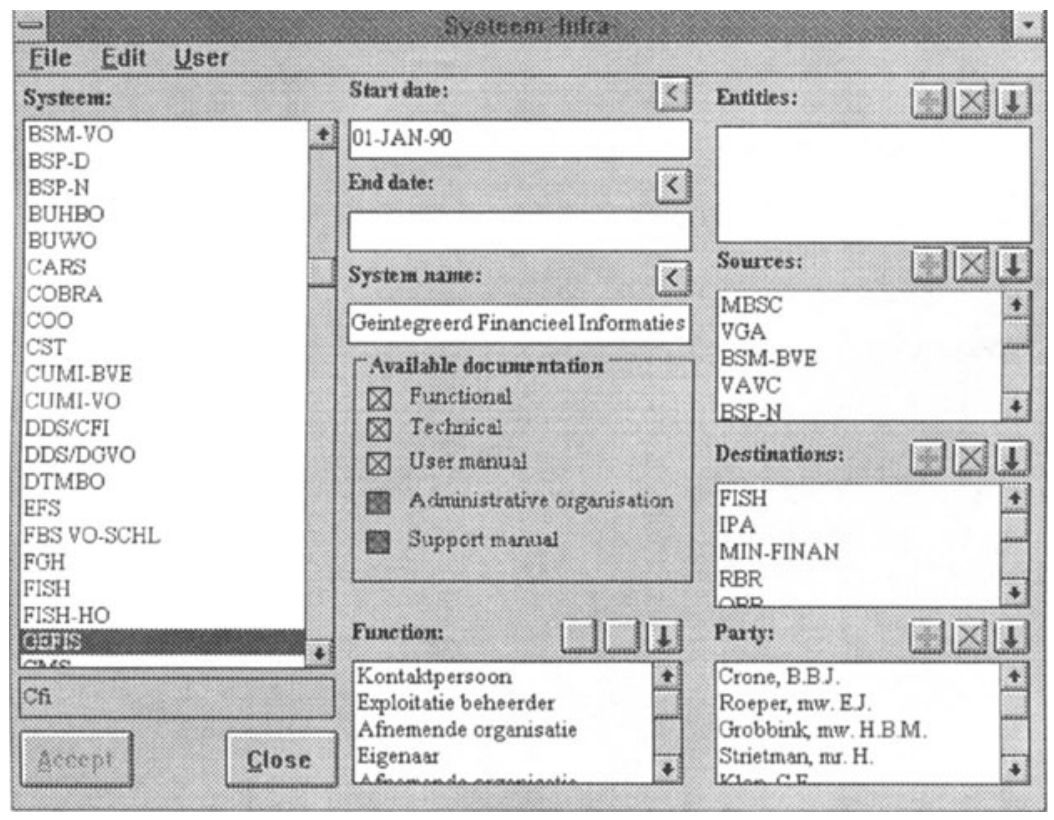

Figure 7 A browser.

As stated before, a browser always operates on one specific object type. At the left side of the browser window, a list of instances of this specific object type is shown. This list contains only the set of instances which are within the scope defined before. At any time, one of the objects in the object list is the current object, as shown by the selection bar in the list. The remaining fields and lists of the browser show the attribute values and relations of the current object.

Attribute values can be edited by simply clicking and typing in the appropriate field. For relations, buttons are provided to add, remove or modify related objects. The user can 
navigate through the contents of the environment by double-clicking in relation lists. A new browser is opened after clicking a related object, revealing more information about that object.

\section{Querying}

The type of search process sketched above, i.e. navigating through objects displayed in the browsers, is intended mainly for retrieving information or documentation about an already selected object. The query mechanism in the prototype enables the user to find new objects by specifying conditions for them.

The query mechanism does not require new user interface elements in the prototype. Queries are composed and executed from within the same browser that is used for displaying information about objects. After the user has composed a query, the browser automatically restricts the set of objects displayed to those that satify the conditions specified in the query. Figure 8 shows the same browser as figure 7 , now showing the composition of a query

Conditions on relations are composed by clicking on a number of objects to which the main object has to be related. Conditions on attribute values are entered using a simple expression language. This language offers the usual comparison operators, that can be used to compare the attribute to a value or another attribute.

This concludes the discussion of the functionality offered by the prototype. Reporting is an important aspect of the support environment that is to be implemented yet. Such functionality could be added by offering the possibility to display or print a selection of attributes and relations of the objects resulting from a query.

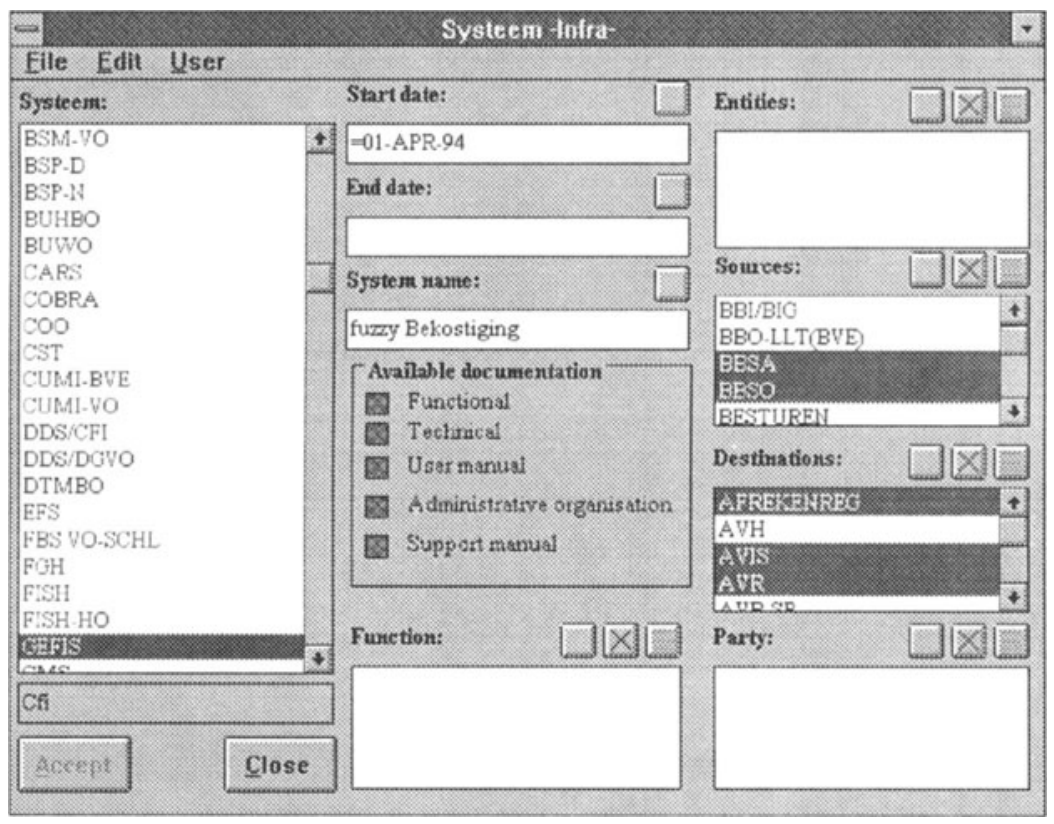

Figure 8 Composing a query. 


\section{Contents of the Environment}

The functionality of the prototype created has been evaluated by filling it with three different types of systems documentation. We have selected documentation from the following three sources:

- SDW. SDW (System Development Workbench), is the leading CASE-tool in The Netherlands. Based on a general repository, this CASE-environment offers different modules, supporting different techniques for systems analysis, design and documentation. From the repository, we selected the documentation of three smaller systems used in the target organisation. The documentation was created originally using the DFD (data flow diagram) and ERD (entity relationship diagram) techniques as supported by SDW.

- Oracle. From the dictionary of the Oracle RDBMS, we took table definitions, with associated packed procedures, of a large registration system used in the target organisation.

- APA. APA stands for A Parameterisable Application. APA is a customisable Oracle application to support one of the main tasks of the organisation, i.e. funding other institutions. This funding business process is dependent on ever changing rules and regulations. APA is capable of calculating the correct funds for these institutions based on a formal representation of the current calculation rules.

The first task in including the data from these three sources in the prototype has been to create a meta-model for each source. However, in order to really maximise the potential reuse, we also developed an abstract meta-model that is capable of handling all three types of systems documentation in a single format. As it has been discussed in section 2 , that last model must be abstract enough to contain the different types of information, which are mutually very differing, yet still be concrete enough to offer sufficient information for developers looking for reusable components.

Because of the flexibility of the prototype of the environment, the abstract model could be included in the environment at the same time as the concrete models for the three types of

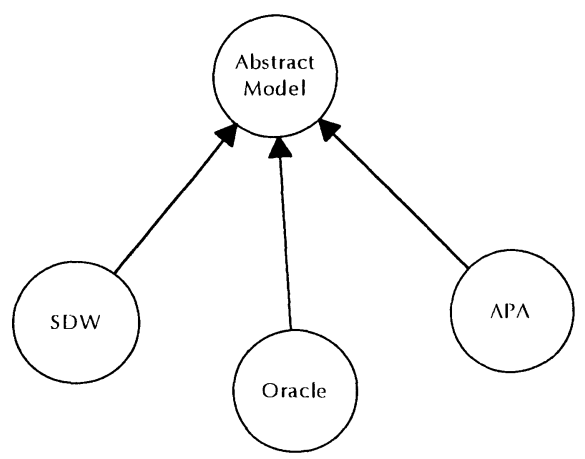

Figure 9 Abstract and concrete models of systems documentation. 
systems documentation. This is illustrated in figure 9. In this way, the information stored can be used to enable reuse across different groups or projects in the target organisation, while at the same time satisfying specific needs of developers working in different implementation platforms.

The construction of the abstract meta-model has been an independent sub-activity in the project (Verhoef, 1995). A discussion of the complete model is beyond the scope of this paper. Here, we discuss its principal structure and the way the three types of systems documentation have been mapped onto this meta-model. A simplified version of the abstract meta-model discussed below is illustrated in figure 10.

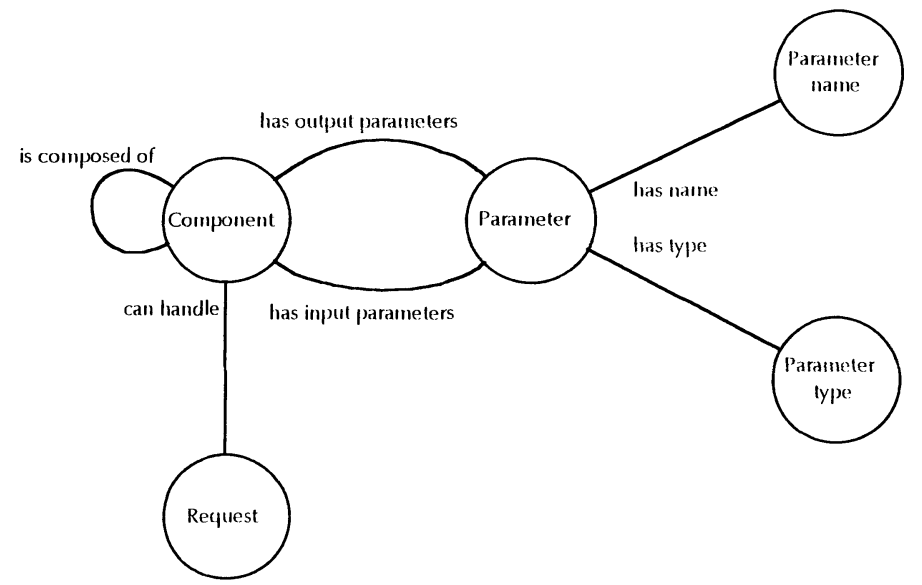

Figure 10 The meta-model of components.

In its essence, the meta-model consists of the object types component and parameter. Two relations are identified between components and parameters. For each component the input and output parameters are kept track of. Components can be selected for reuse on the basis of attributes such as 'application domain', 'implementation platform' and 'basic functionality' that describe the functionality of the component both formally and informally. A parameter has a parameter name and a parameter type.

Components can be constructed using other components. The exact composition of a component is expressed using a formal construction language. A construction expression is parsed to populate the 'is composed of' relation that is included in the meta-model (and therefore is stored in the OMS).

One more concept of the meta-model discussed here is the object type request. A request corresponds to a single unit of basic functionality of a component, similar to a procedure in a module or a query to a database handler. For each request, it is recorded which input parameters are needed. It is also registered which output parameters are the result of the request after the component has finished its activity. 


\section{Epilogue}

We have discussed how general requirements for the disclosure of variable and distributed systems documentation can be translated into a small but powerful number of concrete concepts.

The actual prototype showed the practical significance of the concepts underlying the envisioned support environment. The object management system, based on object types, is flexible enough to capture the different forms of systems documentation. Views allow the usage of several meta-models at the same time in a controlled way. The concept of context can be used efficiently to distribute systems documentation.

Experiments with diverging types of systems documentation have shown that with the prototype based on these concepts, it is possible to support co-ordination and flexibility in document management without losing too much information due to abstract meta-models. That is, the different types of systems documentation are stored in a uniform manner, while still being of practical use to the developer that wishes to reuse components of the systems documented.

The prototype as it is, however, can be improved on several points. At the functional level, capabilities for printing reports lack. As for the implementation, the prototype is not optimised for speed. Especially the object management system can be optimised much further in both time and space usage. However, the prototype did a good job in clarifying the way systems documentation environments could look like. The result will be valuable input for the actual further development of the support environment.

More challenging than producing a smaller and faster environment is its introduction in the target organisation. An organisation with distributed, heterogeneous documentation that aims at maximising reuse differs well from a classical organisation, in which wheels are reinvented everyday and, worse, are redocumented in many different languages. Practical lessons learned from using the prototype in actual development projects will be valuable in this reorganisation process.

\section{References}

Arnold, S.P. and Stepoway, S.L. (1987) The reuse system: cataloguing and retrieval of reusable software, in Software Reuse: Emerging Technology (ed. W. Tracz), 129-137.

Biggerstaff, T. and Perlis, A. Software Reusability, ACM Press, 1989.

Brorsson, M. and Kruzela, I. (1991) Museion-a reuse support system for design of service features, in Proc. of the 10th Anmual International Phoenix Conference on Computers and Communications, IEEE Computer Society, Scottsdale, Arizona.

Burton, B.A., Wienk, R.A., Bailey, S.A., et al. (1987) The reusable software library, IEEE Software, 4 (7).

Constantopoulos, P. and Pataki, E. (1992) A Browser for Software Reuse, in Proc. of the th Int. Conference on Advanced Information Systems CAiSE'92 (ed. P. Loucopoulos), Manchester, UK, Lecture Notes in Computer Science no. 593, Springer-Verlag, 304-326.

Freitag, B. (1994) A hypertext-based tool for large scale software reuse, in Proc. of the 6th Int. Conference on Advanced Information Systems CAiSE'94 (ed. G.M. Wijers), Utrecht, The Netherlands, Lecture Notes in Computer Science no. 881, Springer-Verlag, 283-296. 
Garg, P.K. and Scacchi, W. (1990) A hypertext system to manage software life-cycle documents, IEEE Software, 7 (5).

Helm, R. and Maarek, Y. S. (1990) Integrating information retrieval and domain specific approaches for browsing and retrieval in object-oriented class libraries, OOPSLA '91, 47-61.

Oosterom, N.E. van, Florijn, G., Hofstede, A. ter, et al. (1993) Gegevensdefinitiebeheer in een gedecentraliseerde organisatie, Informatie 35 (2), 128-139 (in Dutch).

Verhoef, T.F. and van der Weide, Th. P. (1995) Gerichte ondersteuning roor systeemontwikkeling met bouwstenen, Note CSI-N9501, University of Nijmegen, The Netherlands (in Dutch).

\section{Biography}

\section{Dr. Duco H. Fijma}

Duco Fijma is a research consultant at ID Research. In 1994, he obtained his Ph.D. degree at the University of Twente, The Netherlands. The central theme in both his Ph.D. research and his current activities is the theory and practice of programming languages and environments. A particular interest within this area is formed by techniques for the (automatic) analysis of both programs and documentation, as applied in, for example, reverse engineering and system renovation. In the project covered by this paper, he concentrated on the design and implementation of the prototype of the support environment discussed.

\section{Dr. T.F. Verhoef}

Denis Verhoef has obtained his Ph.D. degree at Delft University of Technology, in 1993. His $\mathrm{Ph}$.D. study has covered the diversity of information modelling practices, and, consequently, the adaptability of support environments to those different practices. Currently, he is employed as a research consultant at the company ID Research. Particular interests include conceptual modelling, workflow, and Euromethod. Within the project described in this paper, his particular focus has been the development of an abstract meta-model for software components, and the mapping of specific systems documentation types onto that meta-model.

\section{Dr. Gerard M. Wijers}

Dr. Gerard M. Wijers is director of ID Research, Leusden, The Netherlands. In 1991 he finished his PhD study on method engineering and adaptable CASE technology (with honour). For a number of years he has worked at the Software Engineering Research Centre, The Netherlands, as project manager 'Methods and Tools'.

ID Research is an independent consultancy firm for large IT departments on IT-innovation and research. ID Research is specialised in customer specific market surveys, selection procedures and pilot and prototyping projects. Dr. Wijers is Technical Co-ordinator within the Euromethod Programme Management Board. His personal interests are IS development methods, CASE, software quality and metrics, object-orientation and method engineering. He has published many national and international articles and books. 